\title{
Effects of Salinity Acclimation on Histological Characteristics and miRNA Expression Profiles of Scales in Juvenile Rainbow Trout (Oncorhynchus Mykiss)
}

\author{
QiLing Zhou \\ Guangdong Ocean University \\ LiuYong Wang \\ Guangdong Ocean University \\ XiaoLong Zhao \\ Guangdong Ocean University \\ YunSheng Yang \\ Guangdong Ocean University \\ Qian Ma ( $\square$ mfm_0624@163.com ) \\ Guangdong Ocean University \\ Gang Chen \\ Guangdong Ocean University
}

Research Article

Keywords: Oncorhynchus mykiss, scale, salinity acclimation, bone homeostasis, microRNA, histology

Posted Date: January 27th, 2022

DOI: https://doi.org/10.21203/rs.3.rs-1261236/v1

License: () (1) This work is licensed under a Creative Commons Attribution 4.0 International License. Read Full License

Version of Record: A version of this preprint was published at BMC Genomics on April 12th, 2022. See the published version at https://doi.org/10.1186/s12864-022-08531-7. 


\section{Abstract}

Background: The scales serve as an ideal model for studying the regulatory mechanism of bone homeostasis in fish. To explore the effect of salinity acclimation on bone metabolism of juvenile rainbow trout (Oncorhynchus mykiss), three sampling time points during salinity acclimation (7D, 14D and 21D) were selected to detect variations in histological characteristics. In the histological analysis, osteoblast marker enzymes alkaline phosphatase (ALP), osteoclast marker tartrate-resistant acid phosphatase (TRAcP) and calcium salt deposit areas (Von Kossa's) were detected. Changes in calcium (Ca), phosphorus $(P)$ and the molar mass ratio of calcium to phosphorus $(\mathrm{Ca} / \mathrm{P})$ in the scales were also detected by Inductively Coupled Plasma Mass Spectrometry (ICP-MS). In addition, the global MicroRNA (miRNA) expression profiles during salinity acclimation were examined using Illumina sequencing platform because of their important regulatory roles in teleost biological processes.

Results: Twelve independent miRNA libraries were constructed, a total of 664 known and 92 putative novel miRNAs were identified. A total of 290 DEC miRNAs (differentially expressed miRNAs from clusters with significant trends in the cluster analysis) with five types of clustering patterns were obtained, and 5,957 DEC mRNAs (differentially expressed target genes of 22,370 DEC miRNA showed a significant trend in cluster analysis) with six types of clustering patterns were screened according to transcriptome sequencing data. To better understand the functions of the DE miRNAs, 5,957 DEC mRNAs were used for GO and KEGG analysis, and they were significantly enriched in bone metabolism related signaling pathways such as MAPK signaling pathway, Calcium signaling pathway, Wnt signaling pathway, Mineral absorption and NF-kappa B signaling pathway. Six DE miRNAs were randomly selected and their expression were verified by quantitative real-time PCR (qRT-PCR), the expression trends were consistent with the results of transcriptome sequencing.

Conclusions: The DEC miRNAs identified in this study might play an important role in regulation of bone metabolism during salinity acclimation, relative genes or pathways could serve as key candidates for further studies to elucidate molecular mechanism of teleost bone metabolism, and help performing salinity acclimation and developing marine culture of salmonid species.

\section{Background}

Rainbow trout (Oncorhynchus mykiss) is a landlocked, cold-water salmonid species [1]. It has long been considered as one of the excellent breeding species, and is recommended by the FAO (Food and Agriculture Organization of the United Nations) worldwide. As reported, this species can develop and grow normally in seawater [2]. This advantage has long been focused since the adaptation to salinity is closely related to the development, quality and timing of transfer of the fish into seawater for aquaculture [3,4]. Despite the efforts to understand how 0 . mykiss cope with different salinities (osmoregulation mechanism), not much consideration has been given to how the other systems (such as skeletal system) might respond to the salinity adaptation.

Fish skeleton (bones and cartilage) is intimately linked to muscle growth, and is essential for multiple physiological functions such as development, locomotion and load bearing [5]. Similar to the other vertebrates, fish skeleton represents a reservoir of ions such as calcium (Ca), phosphorus (P), and etc., which are in a state of continual exchange with electrolytes found in blood and extracellular fluids [6]. As a complex metabolically active tissue, fish bones undergo continuous remodeling throughout their life. More importantly, bone also plays an important role in plasma ion homeostasis. Previous researches have also demonstrated the importance of linking physiology to biomineral processes [7, 8]. However, the potentially impact of salinity change on bone homeostasis in 0 . mykiss is unclear.

Morphologically, fish bones consist of internal skeleton and exoskeleton. As one of the most important exoskeleton, scales are aligned in partly overlapping rows to protect the fish body. As reported, the scales and skin serve as a barrier to water and ion movements [9]. The removal of scales from just $10 \%$ of the body surface would result in disturbing plasma osmolality and sodium levels in juveniles of some teleost species such as Salmo salar and Oncorhynchus tshawytscha $[10,11]$. In addition, scales are easily accessible dermal bone plates that may be useful models in bone research [12]. As reported, calcium management for the creation of new bony materials and the reorganisation and remobilization of $\mathrm{Ca}$ is present in scales [13]. In other words, a remobilisation of $\mathrm{Ca}^{2+}$ can be found in scales and other bone-like structures. As for 0 . mykiss, scales serve as the first source of Ca remobilisation followed by fins and bony tissue [14]. This indicates that scales may be the best choice to analyze the regulatory mechanism of bone homeostasis in this species. In addition, resorption of teleost scales has been suggested to be initiated under various physiological and experimental conditions [15]. Considerable interest underlying this subject is to establish the molecular mechanisms of the salinity response of bone homeostasis in fish. However, the aforementioned subjects have not been well studied previously in teleost species.

Normally, the bone metabolism is referred as the modeling and remodeling processes (i.e., the actions of osteocytes, osteoclasts, and osteoblasts) in regulating calcium homeostasis [16]. The influence of salinity on the physiology of bone could be analyzed by measuring the enzymatic activity of alkaline phosphatase (ALP) and tartrate-resistant acid phosphatase (TRACP), which could respectively represent the osteoblast and osteoclast activity [17-19]. In addition, Ca content could also reflect calcium homeostasis of bone. As reported, precipitation of calcium phosphates was found to be restricted to the outer (episquamal) layer of the elasmoid scale and could be presented by Von Kossa's staining [20]. Recently, Inductively Coupled Plasma Mass Spectrometry (ICPMS) has also been used to detect the content of $\mathrm{Ca}$ and $\mathrm{P}$ in scales [20,21]. To assess whether bone homeostasis in 0 . mykiss scales is affected by salinity changes, the present study examines the effects of a long-term (21-day) exposure of juvenile 0 . mykiss to sea water, with a specific focus on the impact of salinity on scale miRNA expression profiles.

MicroRNAs (miRNAs) are small, non-coding RNA molecules that regulate gene expression at the post-transcriptional level [22-24]. To date, numerous miRNAs have been discovered in variety of organisms including fish, and the action of these RNAs (with a length of 18-28 nucleotides) was shown to be related to development, homeostasis and many other biological processes [24]. With the application and development of high-throughput technologies, the identification of miRNAs in fish has been widely reported; the studies of these miRNAs have provided new insights into biology [25-27], genome organization, evolution [28], etc. However, the roles of miRNAs in regulating bone homeostasis to environmental change is still unclear. Here, the global miRNA expression profiles in 0 . mykiss scales were studied using Illumina sequencing platform, the differentially expressed miRNAs (DE miRNAs) were characterized to identify novel Ca

Page 2/15 
regulatory or bone homeostasis factors. The results can provide basic information on the molecular signaling pathways involved in bone homeostasis of teleost, and be an important source of information to clarify whether salinity changes could influence the molecular response of bone homeostasis in 0 . mykiss.

\section{Materials And Methods}

\section{Preparation and management of 0 . mykiss}

Juveniles of $O$. mykiss (body weight $46.9 \pm 7.9 \mathrm{~g}$ and body length $15.8 \pm 1.4 \mathrm{~cm}$ ) were collected at Linqu Hatchery Station (Shandong, China) and the experiment was carried out at the same place. A total of 90 fish were equally separated into three groups, each group was domesticated in a flat bottom FRP tank with an effective volume of $1000 \mathrm{~L}$ under a $12 \mathrm{~h}$ light: $12 \mathrm{~h}$ dark photoperiod for seven days (d) prior to the beginning of the experiment. At the start of the experiment, samples were collected as control group (CG) while fish remained at freshwater (3 ppt). Salinity change commenced thereafter by adding seawater into the inflowing water to each tank, salinity was gradually increased at a rate of $4 \mathrm{ppt} / \mathrm{d}$ for a six-day period, and then the fish were maintained in this salinity (27 ppt) afterwards. Scale samples were collected from three individual fish at the time points of 7 days (7D), 14 days (14D) and 21 days (21D).

\section{Sample collection}

Fish were anesthetised using MS-222 (3-Aminobenzoic acid ethyl ester methanesulfonate) to minimize suffering and then scales were collected. A standardised area on the left flank of the fish (extending from the posterior base of the dorsal fin to the base of the caudal fin) was chosen to collect scales of identical size and morphological characters. A total of approximately 100 scales per fish were collected with forceps, eighteen of which were used for histomorphometry analysis, eight were used for ICP-MS, and the rest were used for RNA sequencing (RNA-Seq) and quantitative real-time PCR (qRT-PCR).

\section{Histochemistry and morphometric analysis}

For whole mount studies, scales were fixed in $4 \%$ paraformaldehyde in phosphate buffered saline (PBS, pH $=7.8$ ) at $4{ }^{\circ} \mathrm{C}$ for $24 \mathrm{~h}$. Subsequently, three different kinds of staining experiments were respectively carried out on the scales of 0 . mykiss from four sample groups (CG, 7D, 14D and 21D). Each group was consisting of three fish in parallel, and six scales of each fish were used in parallel for dyeing. Scales were stained for activity of TRAcP and ALP, according to the instruction of Tartrate-Resistant Acid Phosphata stain Kit (D023-1) and BCIP/NBT stain Kit (I023-1) (Nanjing Jiancheng Bioengineering Institute, Nanjing, China) respectively.

In addition, samples were stained for presence of calcium phosphates using Von Kossa's Kit (G3282) (Beijing Solarbio Science \& Technology Co., Ltd., Beijing, China), following the manufacturer's instructions. Breifly, scales were incubated with $5 \%$ silver nitrate under bright light for $1 \mathrm{~h}$, subsequently rinsed with tap water for $1 \mathrm{~min}$, developed for $5 \mathrm{~min}$ in $5 \%$ sodium thiosulfate, and finally rinsed thoroughly with tap water.

\section{Calcium and phosphorus analysis}

Eight ontogenetic scales of each fish were washed with ultrapure water, dried, dissolved in 200 ul nitric acid (65\%), and then treated with microwave digestion apparatus (Anton Paar Multiwave Pro 41HVT56). The obtained individual samples were respectively diluted 100 times with ultrapure water, the content of Ca and $\mathrm{P}$ of each sample was measured by ICP-MS (Agilent $7500 \mathrm{CX}$ ), and the $\mathrm{Ca} / \mathrm{P}$ was then calculated.

\section{RNA extraction, library construction, and sequencing}

Total RNA was respectively isolated from 0 . mykiss scale samples at CG, 7D, 14D and 21D group using TRIzol reagent (Invitrogen, CA, USA) according to the manufacturer's procedure. The quality of total RNA was analyzed by Bioanalyzer 2100 (Agilent Technologies, Santa Clara, CA, USA), and the concentration and the quality of the total RNA was analyzed using the NanoDrop 2000 (Thermo Fisher Scientific, Lafayette, CO, USA). Twelve small RNA libraries were constructed following the procedure from TruSeq Small RNA Sample Prep Kits (Illumina, San Diego, USA). The libraries were then single-end 50 bp sequenced using an Illumina Hiseq 2000/2500 platform (Lc-bio, Hangzhou, China). For the transcriptome sequencing, a chain-specific library was constructed by rRNA depletion. After the library was qualified, Illumina Hiseq 4000 was used for sequencing, and the sequencing read length was $2 * 150$ bp (PE 150 ).

\section{Identification of miRNAs}

The miRNA sequences were identified by ACGT101-miR (LC Sciences, Houston, Texas, USA). In briefly, adapter dimers, junk, low complexity, common RNA families (rRNA, tRNA, snRNA, snoRNA) and repeats in raw reads were removed. Subsequently, unique sequences with a length of 18 26 nucleotide were mapped to specific species precursors in miRBase 22.0 by BLAST search to identify known miRNAs and novel 3p-and 5p-derived miRNAs. The unmapped sequences were BLASTed against the specific genomes, and the hairpin RNA structures containing sequences were predicated from the flank 80 nt sequences using RNAfold software (http://rna.tbi.univie.ac. at/cgi-bin/RNAfold.cgi).

\section{Transcriptome sequencing and analysis}

Using the cutadpter software, the joint reads and the low-quality data reads in the raw data were removed to obtain the valid data reads. The obtained reads were assembled and quantified using the transcript assembly software StringTie. Then, the expression level for mRNAs were performed by calculating fragments per kilobase of exon per million reads mapped (FPKM). Finally, the obtained gene and their functional annotation after assembly were compared with the protein sequences in the five public databases (Swiss-Prot, NR, KEGG, KOG and Pfam).

Prediction and enrichment analysis of genes targeted by miRNAs 
To identify DE transcripts across samples or groups, the edgeR package was used. We identified miRNA with a fold change $\geq 2$ and a false discovery rate $(F D R)<0.05$ in a comparison as DE miRNAs. To predict target genes (mRNAs) from all known and novel DEC miRNAs, two computational target prediction algorithms (TargetScan 50 and Miranda 3.3a) were used to identify miRNA binding sites. And the parameters of TargetScan were set as more than or equal to 90 , the parameters of Miranda were set as less than $-20 \mathrm{kcal} / \mathrm{mol}$. Finally, the data predicted by both algorithms were combined and the overlaps were calculated. The GO terms and KEGG pathway of these most abundant miRNAs, miRNA target genes were also annotated.

\section{Express trend analysis of miRNAs and their target genes}

Short Time-series Expression Miner (STEM, version1.2.2b) software was used to perform trend cluster analysis of the miRNAs and target genes expression patterns in four groups.

\section{Validation of miRNA expression by qRT-PCR}

Expression of six randomly selected miRNAs was detected using qRT-PCR. The primers used are shown in Table 1. The cDNA was synthesized using the miRNA 1st strand cDNA synthesis kit with gDNA eraser (Accurate biology, Changsha, China) from $1 \mu \mathrm{g}$ total RNA according to the manufacturer's instructions. qRT-PCR was performed at a final volume of $20 \mu$ using SYBR ${ }^{\circledR}$ Green Premix Pro Taq HS qPCR Kit II (Accurate biology, Changsha, China) with the LightCycler 96 (Roche, $\mathrm{CHE}$ ). The abundance of each selected miRNA was normalized to the level of the U6 small nuclear RNA (snRNA). The ratio change of each miRNA was determined by $2^{-\triangle \triangle C T}$ method. All reactions were performed in triplicate.

\section{Statistical analysis}

The statistical analyses were performed in SPSS 20.0. Data are expressed as the mean \pm SD format. Student's $t$-test was selected to evaluate differences between two groups in qRT-PCR. A $P$-value $<0.05$ was considered to indicate a significant difference.

\section{Results}

\section{Histochemistry and morphometric analysis}

TRAcP staining of scales

The results of TRACP staining on the scales of juvenile 0 . mykiss were shown in Fig. 1, the positive TRAcP sites were red and mainly located at the scale margins. As for the CG scales, the area of positive TRAcP staining sites was small. Compared with CG scales, the TRAcP positive staining area and the number of positive reaction sites of the scales in 7D and 21D group were larger.

\section{ALP staining of scales}

The results of ALP staining on the scales of juvenile 0 . mykiss were shown in Fig. 2, and the ALP positive sites were stained purple. A large positive staining area was found in the middle of the scales, and no significant difference was identified in the scales of different groups.

\section{Von Kossa's staining of scales}

The results of Von Kossa's staining on the scales of juvenile 0 . mykiss were shown in Fig. 3, and the positive sites of calcium salts were stained brown. A large area of positive Von Kossa's staining was identified in the middle of the scales. The positive Von Kossa's staining in 7D scales was stronger than scales of the other three groups. No resorption pit was observed on scales of all the four groups. The location of Von Kossa's staining area was similar to that of the ALP staining area, and the calcium salt deposits less in the TRAcP positive staining area.

\section{Calcium and phosphorus analysis of scales}

Changes in Ca content of the scales throughout the experimental period were shown in Fig. 4 (A), and the result was consistent with the Von Kossa's staining results. The Ca content first increased and reached the highest level $(0.100 \mu \mathrm{mol} / \mathrm{scale})$ at $7 \mathrm{D}$. The Ca content of the scales at 7D, 14D and 21D were significantly higher than that of the CG scales $(P$-value $<0.05)$. The P content of the scales [Fig. 4 (B)] also reached the highest level $(0.104 \mu \mathrm{mol} / \mathrm{scale})$ at $7 \mathrm{D}$, which was significantly higher than that of the $\mathrm{CG}$ scales $(0.081 \mu \mathrm{mol} / \mathrm{scale})(P$-value $<0.05)$; the $14 \mathrm{D}$ scales exhibited the lowest $\mathrm{P}$ content $(0.067$ $\mu \mathrm{mol} / \mathrm{scale})(P$-value $<0.05)$.

The molar mass ratio of calcium to phosphorus $(\mathrm{Ca} / \mathrm{P})$, indicating the crystalline phase of calcium phosphate (e.g., pure calcium hydroxyapatite, theoretical ratio $=1.67$ ), was also shown in Fig. $4(\mathrm{C})$. The $\mathrm{Ca} / \mathrm{P}$ of $14 \mathrm{D}$ scales was significantly higher than scales of the other three groups, and no significant difference was found among $\mathrm{Ca} / \mathrm{P}$ of $\mathrm{CG}, 7 \mathrm{D}$ and $21 \mathrm{D}$ scales.

\section{Illumina sequencing of miRNAs in 0. mykiss}

To identify miRNAs in the scales of juvenile O. mykiss, twelve miRNA libraries from CG (CG_1, CG_2 and CG_3), 7D (7D_1, 7D_2 and 7D_3), 14D (14D_1, 14D_2 and 14D_3) and 21D (21D_1, 21D_2 and 21D_3) were respectively constructed and sequenced using Illumina sequencing technology. A total number of $11,277,023,11,052,110,12,969,211$ and $11,929,493$ raw reads were respectively obtained from CG, 7D, 14D and 21D scales. After preprocessing steps, a total of $921,415,824,625,946,666$ and 894,351 clean reads were obtained due to the removal of low-quality reads, adapters et al. The aforementioned reads respectively represented $341,328,261,252,245,627$ and 236,173 unique sequences (valid reads) (Table 2). Furthermore, the length distribution analysis of these miRNA sequences showed a similar pattern of distribution in length of all libraries (Fig. 5). The length of the miRNAs in all libraries varied from 18 to 26 
nt and the majority of read length was 22 and $23 \mathrm{nt}$, followed by 21, 24, 25 and $26 \mathrm{nt}$. The read length of $22 \mathrm{nt}$ in the four groups respectively accounted for $23.31 \%, 24.75 \%, 33.40 \%$ and $35.18 \%$ of the total miRNA numbers. The raw reads of the twelve libraries were uploaded into the NCBI database Sequence Read Archive (SRA) and the SRR numbers were from SRR15559621 to SRR15559632.

Table 1 Primer sequences used for qRT-PCR

\begin{tabular}{|ll|}
\hline Primer name & Primer sequence $\left(5^{\prime} \sim 3^{\prime}\right)$ \\
\hline DRE-MIR-193B-3P-F & CCCGCAAAGTCCCGCTAAA \\
\hline DRE-MIR-193B-3P-R & CAGTGCAGGGTCCGAGGTAT \\
\hline SSA-MIR-203A-3P-F & GTGAAATGTTTAGGACCACTTG \\
\hline SSA-MIR-203A-3P-R & CAGTGCAGGGTCCGAGGTAT \\
\hline SSA-MIR-1338-3P-F & TCAGGTTCGTCAGCCCATG \\
\hline SSA-MIR-1338-3P-R & CAGTGCAGGGTCCGAGGTAT \\
\hline SSA-MIR-92A-3P-F & TATTGCACTTGTCCCGGCCT \\
\hline SSA-MIR-92A-3P-R & CAGTGCAGGGTCCGAGGTAT \\
SSA-MIR-205A-5P-F & TTCCTTCATTCCACCGGATC \\
\hline SSA-MIR-205A-5P-R & CAGTGCAGGGTCCGAGGTAT \\
\hline ONI-MIR-125A-F & CCTGAGACCCTTAACCTGTG \\
\hline ONI-MIR-125A-R & CAGTGCAGGGTCCGAGGTAT \\
\hline U6-F & CTCGCTTCGGCAGCACATATACT \\
\hline U6-R & ACGCTTCACGAATTTGCGTGTC \\
\hline
\end{tabular}

Table 2Analysis of miRNA sequences of juvenile O. mykiss scales collected at different time points during salinity acclimation

\begin{tabular}{|c|c|c|c|c|c|c|c|c|c|c|c|c|c|}
\hline \multirow[t]{2}{*}{ Type } & \multicolumn{4}{|l|}{ CG } & \multicolumn{4}{|l|}{ 7D } & \multicolumn{4}{|l|}{$14 \mathrm{D}$} & 2 \\
\hline & Total & $\%$ & unique & $\%$ & Total & $\%$ & unique & $\%$ & Total & $\%$ & unique & $\%$ & 7 \\
\hline $\begin{array}{l}\text { Raw } \\
\text { reads }\end{array}$ & $11,277,023$ & 100.00 & 921,415 & 100.00 & $11,052,110$ & 100.00 & 824,625 & 100.00 & $12,969,211$ & 100.00 & 946,666 & 100.00 & 1 \\
\hline $\begin{array}{l}\text { 3ADT\& } \\
\text { length } \\
\text { filter }\end{array}$ & $5,251,562$ & 46.50 & 567,743 & 61.85 & $5,157,869$ & 45.88 & 552,577 & 66.90 & $6,958,124$ & 53.57 & 689,174 & 72.84 & $t$ \\
\hline $\begin{array}{l}\text { Junk } \\
\text { reads }\end{array}$ & 30,525 & 0.27 & 3,428 & 0.37 & 23,126 & 0.21 & 2,505 & 0.30 & 9,056 & 0.07 & 2,208 & 0.23 & $\varepsilon$ \\
\hline Rfam & 542,449 & 4.83 & 7,238 & 0.79 & 564,990 & 5.15 & 7,133 & 0.88 & 509,731 & 3.94 & 8,623 & 0.92 & $t$ \\
\hline mRNA & 41,515 & 0.37 & 1,721 & 0.18 & 33,869 & 0.32 & 1,167 & 0.14 & 46,402 & 0.36 & 1,114 & 0.12 & $t$ \\
\hline Repeats & 109,582 & 0.98 & 517 & 0.06 & 154,111 & 1.37 & 481 & 0.06 & 66,809 & 0.51 & 787 & 0.08 & ; \\
\hline $\begin{array}{l}\text { valid } \\
\text { reads }\end{array}$ & $5,412,133$ & 48.04 & 341,328 & 36.81 & $5,272,049$ & 48.45 & 261,252 & 31.77 & $5,447,228$ & 42.07 & 245,627 & 25.90 & $\angle$ \\
\hline
\end{tabular}

Identification and specific expression of miRNAs in O. mykiss scales

To identify miRNAs in the scales of juvenile 0 . mykiss, all valid sequences were compared with known vertebrate miRNAs and miRNA precursor sequences in miRBase database. As a result, a total of 756 ( 664 known and 92 putative novel miRNAs) miRNAs were identified (Table S1). The read numbers of these known miRNAs ranged from 1 to 483,390, indicating that there were significantly difference in expression levels of different miRNAs. These miRNAs covered 121 miRNA families, and the most abundant families were let-7 (40 members), miR-10 (29 members) and miR-30 (24 members) (Table S2).

In the 756 miRNAs, 514 miRNAs showed co-expressed in all the four groups (Fig. 6). Eight,18, 29 and 49 miRNAs were uniquely expressed in CG, 7D, 14D and 21D scales, respectively. In all the four groups, 21D scales exhibited the largest number of unique miRNAs. 594 miRNAs were expressed in scales of CG, 607 were expressed in 7D, 636 were expressed in 14D, and 645 were expressed in 21D.

\section{Transcriptome sequencing data}

RNA-Seq was performed in juvenile O. mykiss scales of each sample, and a total of $147.29 \mathrm{~Gb}$ valid data was obtained. Quality of the RNA-Seq data and comparison of mRNA sequences from different groups were shown in Table 3 . The valid data of each sample reached $11.22 \mathrm{~Gb}$, and the minimum percentage of Q30 bases was $97.32 \%$. The valid data of each sample was compared with the 0 . mykiss genome, and the minimum comparison efficiency reached $82.74 \%$. 
Table 3Statistics of RNA-seq data from each juvenile O. mykiss scale sample collected at different time points during salinity acclimation

\begin{tabular}{|llllll|}
\hline Sample & Raw Read & Valid Read & Valid data/G & Q30/\% & Mapped reads \\
\hline CG_1 & $89,627,272$ & $83,437,990$ & $12.52 G$ & 98.71 & $72,429,195(86.81 \%)$ \\
\hline CG_2 & $90,637,284$ & $87,198,854$ & $13.08 G$ & 98.58 & $78,893,393(90.48 \%)$ \\
\hline CG_3 & $80,356,512$ & $74,812,354$ & $11.22 G$ & 98.25 & $64,591,320(86.34 \%)$ \\
\hline 7D_1 & $92,595,784$ & $82,794,096$ & $12.42 G$ & 98.59 & $71,541,252(86.41 \%)$ \\
\hline 7D_3 & $95,578,444$ & $89,211,058$ & $13.38 G$ & 98.09 & $76,755,268(86.04 \%)$ \\
\hline 14D_1 & $91,322,682$ & $76,219,276$ & $11.43 G$ & 98.49 & $63,555,106(83.38 \%)$ \\
\hline 14D_2 & $94,608,098$ & $75,339,848$ & $11.30 G$ & 98.58 & $62,339,088(82.74 \%)$ \\
\hline 14D_3 & $90,570,502$ & $80,280,090$ & $12.04 G$ & 98.54 & $67,911,295(84.59 \%)$ \\
\hline 21D_1 & $94,544,622$ & $76,698,318$ & $11.50 G$ & 98.56 & $67,600,190(88.14 \%)$ \\
\hline 21D_2 & $91,295,438$ & $86,198,836$ & $12.93 G$ & 97.37 & $73,723,516(85.53 \%)$ \\
\hline 21D_3 & $88,674,894$ & $85,859,784$ & $12.88 G$ & 97.32 & $72,851,690(84.85 \%)$ \\
\hline
\end{tabular}

\section{Differential expression analysis and target genes prediction of the identified miRNAs}

The trend of miRNA expression at different timepoints (CG, 7D, 14D and 21D) was analyzed using STEM software. As shown in Fig. 7, the 327 DE miRNAs were clustered into 30 expression patterns, of which five expression patterns were with significant changing trends (Cluster 10, Cluster 11, Cluster 17, Cluster 18 and Cluster 25) ( $P$-value < 0.05). A total of 290 DEC miRNAs (DE miRNAs from clusters with significant trends in the cluster analysis, DEC miRNAs) were clustered into these five patterns (contains 22 DEC miRNAs in Cluster10; Cluster 11 contains 37 DEC miRNAs; Cluster 17 contains 91 DEC miRNAs; Cluster 18 contains 70 DEC miRNAs; Cluster 25 contains 70 DEC miRNAs), and detailed information was listed in Table S3. A total of 22,374 predicted target genes of the DEC miRNAs from the aforementioned five expression modes were obtained; the DEC miRNAs in Cluster 10, Cluster 11, Cluster 17, Cluster 18 and Cluster 25 each had $3,897,5,756,9,423,8,586$ and 8,977 predicted mRNAs.

Additionally, trend analysis was performed on 22,374 predicted target genes of DEC miRNAs. The co-clustering was also divided into 30 expression patterns, among which 5,957 DEC mRNAs (DE mRNAs of 22,370 DEC miRNA target genes showed a significant trend in cluster analysis, DEC mRNAs) were clustered into 6 expression patterns with significant changing trends (Cluster 16, Cluster 17, Cluster 22, Cluster 24, Cluster 27 and Cluster 29$)(P$-value < 0.05$)($ Fig. 8$)$. Detailed information was listed in Table S4. Cluster 16 contains 682 DEC mRNAs; Cluster 17 contains 1,140 DEC mRNAs; Cluster 22 contains 387 DEC mRNAs; Cluster 24 contains 1,092 DEC mRNAs; Cluster 27 contains 740 DEC mRNAs; Cluster 29 contains 1,916 DEC mRNAs.

\section{GO analysis of DEC miRNA-targeted genes}

Gene ontology (GO) enrichment (function) analysis was performed on predicted DEC mRNAs to explain and speculate the function of DEC mRNAs (Fig. 9). Detailed information of the GO analysis was listed in Table S5. As shown in Fig. 9, the predicted DEC mRNAs could be classified into three major categories: biological process (BP), cellular component (CC), and molecular function (MF). The columns in each category presented in the figure were in descending order of percent of genes. The DEC mRNAs in the BP category were enriched in regulation of transcription, DNA-templated, phosphorylation, signal transduction, transport, et al. The DEC mRNAs in the CC category were associated with terms such as membrane, integral component of membrane, cytoplasm, nucleus and cytosol. In the MF category, the DEC mRNAs were associated with terms such as metal ion binding, ATP binding, nucleotide binding and transferase activity.

\section{KEGG pathway analysis of DEC miRNA-targeted genes}

Kyoto Encyclopedia of Genes and Genomes (KEGG) analysis was performed to analyze the biological pathways of the predicted DEC mRNAs (Fig. 10). Detailed information of the KEGG pathways ( $P$-value< 0.05 ) was listed in Table S6. predicted DEC mRNAs were significantly enriched in the pathways such as the MAPK signaling pathway, Toll-like receptor signaling pathway, mTOR signaling pathway, Thyroid hormone signaling pathway, Calcium signaling pathway, VEGF signaling pathway, ErbB signaling pathway, Insulin secretion, Wnt signaling pathway, TGF-beta signaling pathway, FoxO signaling pathway, Insulin signaling pathway, Mineral absorption, NF-kappa B (NF-KB) signaling pathway and other bone metabolism related signaling pathways.

\section{Validation of DE miRNAs using qRT-PCR}

A total of six randomly selected miRNAs, including DRE-MIR-193B-3P, SSA-MIR-203A-3P, SSA-MIR-1338-3P, SSA-MIR-92A-3P, SSA-MIR-205A-5P and ONI-MIR125A, were used for qRT-PCR analysis. As shown in Fig. 11, the expression pattern of the six miRNAs were completely identical with the transcriptome sequencing results.

\section{Discussion}

Based on the scale TRAcP staining results of juvenile 0 . mykiss, the number and area of absorption pits was found along the edges of the scales. This location of positive TRAcP staining was similar to that of the zebrafish scales, which showed a detection of TRAcP along the entire length of the grooves of 
the scale and in the focus. Previously, the TRAcP has been used as a marker for osteoclastic activity in 0 . mykiss scales, and it has been reported that the TRAcP activity could be induced by injection of estradiol-17 $\beta[21,29]$. Here in this study, the TRAcP activity in the scales of juvenile $O$. mykiss was also increased along with the salinity treatment and the highest level was detected on the 7D samples, which indicated a significant effect of salinity acclimation on the osteoclast activity. As for the ALP staining results, no significant difference was identified in the activity of osteoblasts of scales from different groups. According to these results, an increase on the absorption of calcium in the scales was anticipated because that the activation of osteoclast always resulted in demineralization. However, the calcium salt deposition of scales was significantly increased along with the salinity acclimation based on the Von Kossa's staining and ICP-MS results. The possible reasons were analyzed as followed: (1) juvenile 0 . mykiss were used for the salinity acclimation, skeleton of fish at the early developmental stages were in the period of rapid growth, and high osteoblast activity was maintained. The effect of salinity acclimation on osteoclasts was not strong enough to exceed the results of the high osteoblast activity. (2) demineralization is a relatively slower process than the change in enzymes or cell activity. Even though salinity acclimation stimulated the absorption of exogenous calcium, it might take time for the unmineralization to process.

In addition to the above-mentioned histochemistry and morphometric changes on the scales, a total of 756 ( 664 known and 92 putative novel miRNAs) miRNAs were identified, and a total of 290 DEC miRNAs were also identified in O. mykiss scale samples collected along with the salinity acclimation. The number of the identified miRNA were larger than those of the skeleton tissues (bone and cartilage) in mammal species. For instance, Desjardin [30] identified miRNAs in equine subchondral bone and cartilage by next-generation sequencing, 622 and 609 miRNAs were respectively identified, including about 300 novel miRNAs, and the results showed that they were involved in the physiopathological process of osteochondrosis. As for the articular cartilage of rats, a total of 310 known miRNAs as well as 86 novel miRNAs candidates were identified, these miRNAs were found to be targeting chondrocyte proliferation and differentiation regulatory factors, such as Wnts, BMPs, Runx2, Sox9, HDAC4, et al. [31]. However, the function of these miRNAs in regulating skeleton development was still unclear.

In order to explore the role of DE miRNAs in the regulation of 0 . mykiss bone metabolism, KEGG enrichment analysis was performed on the target genes of these miRNAs. As a result, a few DE miRNAs were screened out because their target genes were mainly enriched in bone metabolism-related signal pathways. As for the bone homeostasis in teleost, both bone resorption (osteoclast formation and differentiation, osteoblast/chondrocyte apoptosis) and bone formation (osteoblast/chondrocyte formation and differentiation, inhibition of osteoclast) are important processes [16]. Here in this study, some of the mentioned DE miRNAs were related to regulation of promoting osteoclast formation and differentiation or chondrocyte apoptosis. For instance, the target genes of SSA-MIR30B-5P_R-1, SSA-MIR-181A-5P, GMO-MIR-181B-5P_R-1 and GMO-MIR-199-5P_R+1 were significantly enriched in the NF-KB signaling pathway. This pathway has been considered to be a common final pathway for many inflammatory mechanisms in the pathophysiology of cartilage [32]. It has been reported that the activation of NF-KB could induce the secretion of cytokines such as IL-1 $\beta$, IL-18 and TNF- $\alpha$, which would have an effect on cartilage matrix remodeling, chondrocyte apoptosis, synovial inflammation, and cause cartilage damage [33]. Genes in this pathway could also participate in osteoclast formation, differentiation, maturation and anti-apoptosis of vertebrate [32]. As reported, the target gene of SSA-MIR-181A-5P and GMO-MIR-181B-5P_R-1 such as NF-KB inhibitor alpha-like and calreticulin could activate the NF-KB signaling pathway, and ultimately achieve the purpose of promoting osteoclast formation and bone resorption.

In addition, the DE miRNAs tni-miR-181a-5p and ola-miR-181b-5p_R-1 were found to be targeting tlr2, which was an important factor in the Toll-like receptor (TLR) signaling pathway. TLR2 could induce mouse macrophages to secrete TNF-a or activating NF-KB to promote osteoclast formation and bone resorption [34]. Another important signaling pathway is the MAPK signaling pathway. By promoting the overexpression of IL-1 $\beta$ and TNF- $a$, activation of MAPK signaling pathway could lead to cartilage damage in articular of Vertebrate [35]. Significant difference in the expression of dre-miR-181a-5p_L+2 and its target gene IL$1 \beta$ were identified between salinity acclimation and CG scales in this study. Furthermore, the DE miRNAs gmo-miR-23a-3p, tni-miR-23b and ssa-miR-19c-3p were also found to be targeting TNF. These results indicated that these genes might be involved in the apoptosis of chondrocytes [36].

Among the DEC miRNAs, SSA-MIR-26A-5P, ONI-MIR-27B, GMO-MIR-27D-5P_L+1R-2, GMO-MIR-125B-3P_R+3, SSA-MIR-133B-3P_L-1R+2, DRE-MIR-204-5P_R+2, ONI-MIR-206 and other miRNAs target genes were mainly enriched in Wnt, TGF- $\beta$, Notch and other bone metabolism-related signaling pathways. According to Friedlander [37], some genes in the classic Wnt pathway can not only promote the differentiation of mouse osteoblasts, the formation and mineralization of bone matrix. In this study, oni-miR-27e and ssa-miR-29a-3p_R+2_1ss18AG could enhance Wnt signal transduction by down-regulating the negative regulatory gene dkk1 in the Wnt signaling pathway, thereby promoting osteoblast differentiation. It was consistent with the research results of Westendorf [38] in hFOB culture in vitro. It was speculated that oni-miR-27e and ssa-miR-29a-3p_R+2_1ss18AG were important osteoblast differentiation regulating miRNAs. In this study, according to GO and KEGG annotation results, it was speculated that GMO-MIR-125B-3P_R+3 inhibited osteoblast differentiation by targeting bmp-2. $\mathrm{Cbfb}$ gene is an essential cofactor for the transcription factor Runx2 during osteoblast differentiation. Huang [39] found that over-expression of miR-125b in $\mathrm{C} 3 \mathrm{H} 10 \mathrm{~T} 1 / 2$ cells could interfere with the expression and down-regulate Cbfb protein, and over-expression reduced the mRNA levels of three osteogenic marker genes including ALP, osteocalcin (OCN) and osteopontin (OPN) induced by BMP-2, whereas, anti-mir-125b increased the expression of these marker genes and hence up-regulated mRNA levels of Cbfb. In this study, dre-miR-125c-5p_R+1_1ss18CT was also found to play a regulatory role in osteoblast differentiation by targeting cbfb. The results showed that miR-125b was a key regulator of osteoblast differentiation.

\section{Conclusion}

The effect of salinity acclimation on bone metabolism of 0 . mykiss was confirmed based on the histochemistry and morphometric analysis of the scales in CG, 7D, 14D and 21D, showing that osteoclastic activity and the calcium salt deposition of scales were significantly increased. Then the transcriptome analysis of the aforementioned samples was conducted using RNA-seq. Variations in expression patterns of miRNAs were identified, GO and KEGG pathway analysis of the DE miRNAs revealed that they might play important roles in regulation of bone metabolism during salinity acclimation. Our findings provide candidate key genes or pathways for follow-up work to elucidate molecular mechanism of teleost bone metabolism, which could help performing salinity acclimation and developing marine culture of salmonid species. 


\section{Abbreviations}

ALP: alkaline phosphatase; TRAcP: tartrate-resistant acid phosphatase; Ca: calcium; P: phosphorus; Ca/P: molar mass ratio of calcium to phosphorus; ICPMS: Inductively Coupled Plasma Mass Spectrometry; miRNAs: MicroRNAs; snRNA: small nuclear RNA; FAO: Food and Agriculture Organization of the United Nations; MS-222: 3-Aminobenzoic acid ethyl ester methanesulfonate; RNA-seq: RNA sequencing; CG: control group; 7D: 7 days; 14D: 14 days; 21D: 21 days; DEC miRNAs: differentially expressed miRNAs from clusters with significant trends in the cluster analysis; DEC mRNAs: differentially expressed target genes of 22,370 DEC miRNA showed a significant trend in cluster analysis; DE miRNAs: differentially expressed miRNAs; FPKM: fragments per kilobase of exon per million reads mapped; FDR: false discovery rate; STEM: Short Time-series Expression Miner; GO: Gene oncology; KEGG: Kyoto encyclopedia of gene and genomes; BP: biological process; CC: cellular component; MF: molecular function; qRT-PCR: quantitative real-time PCR

\section{Declarations}

\section{a. Ethics approval and consent to participate}

All animal experiments were conducted strictly based on the recommendations in the 'Guide for the Care and Use of Laboratory Animals' set by the National Institutes of Health. The animal protocols and all methods in this study were approved by the Animal Ethics Committee of Guangdong Ocean University (Zhanjiang, China). The study was carried out in compliance with the ARRIVE guidelines.

\section{b. Consent for publication}

Not applicable.

\section{c. Availability of data and materials}

All of the raw data have been uploaded to the NCBI database Sequence Read Archive (SRA) and the SRR numbers were from SRR15559621 to SRR15559632. The data can be accessed at https://www.ncbi.nlm.nih.gov/bioproject/PRJNA753226. The miRNA database we refer to can be found at $\mathrm{ftp}: / /$ mirbase.org/pub/mirbase/CURRENT/, the Genome Database can be found at https://www.ncbi.nlm.nih.gov/genome/?term=0ncorhynchus\%20mykiss, and the mRNA Database can be found at https://www.ncbi.nlm.nih.gov/genome/?term=Oncorhynchus\%20mykiss. All of the datasets referenced in this study can be obtained upon reasonable request to the corresponding authors.

\section{d. Competing interests}

The authors declare that they have no competing interests.

\section{e. Funding}

This study was supported by the National Natural Science Foundation of China [grant number 31772828], Program for Scientific Research Start-up Funds of Guangdong Ocean University [grant number R19022].

\section{f. Authors' contributions}

Qiling Zhou and Qian Ma conceived and designed the research plans, completed the data processing, normalization, and bioinformatics analyses, wrote the article with contributions from all the authors. Qian Ma proposed the conception of the work and substantively revised the manuscript, supervised and corrected the writing. All authors including Liuyong Wang, XiaoLong Zhao, Yunsheng Yang and Gang Chen approved the final submission of this manuscript.

\section{g. Acknowledgments}

We thank all people who have given help or suggestions to this project. We acknowledge the LC-Sciences (Hangzhou, China) for the facilities and expertise of the PacBio platform for libraries construction, sequencing, library construction, and sequencing. We would like to thank Erjun Yang and Feifan Mao for their help in sampling. Thanks to Dr. Yangen Zhou (Ocean University of China) for providing constructive comments.

\section{References}

1. Johnsson J, Clarke WC. Development of seawater adaptation in juvenile steelhead trout (Salmo gairdneri) and domesticated rainbow trout (Salmo gairdneri)-Effects of size, temperature and photoperiod. Aquaculture. 1988;71(3):247-263.

2. Landless PJ. Acclimation of rainbow trout to sea water. Aquaculture. 1976;7(2):173-179.

3. Kendall NW, Mcmillan JR, Sloat MR, et al. Anadromy and residency in steelhead and rainbow trout (Oncorhynchus mykiss): a review of the processes and patterns. Canadian Journal of Fisheries \& Aquatic Sciences. 2015;72(3):1-24.

4. Xiong, YH, Wang X, Dong SL, et al. Comparisons of Salinity Adaptation in Terms of Growth, Body Composition, and Energy Budget in Juveniles of Rainbow and Steelhead Trouts (Oncorhynchus mykiss). Journal of Ocean University of China. 2019;18(2):509-518.

5. Sansom IJ. Bones and cartilage: developmental and evolutionary skeletal biology, by Brian K. Hall. Blackwell Publishing Inc. 2006;8(4):389-390.

6. Lall SP, Lewis-Mccrea LM. Role of nutrients in skeletal metabolism and pathology in fish - An overview. Aquaculture. 2007;267(1-4):3-19.

7. Guerreiro PM. Water calcium concentration modifies whole-body calcium uptake in sea bream larvae during short-term adaptation to altered salinities. Journal of Experimental Biology. 2004;207(4):645-653. 
8. Sturrock AM, Hunter E, Milton JA, et al. Data from: Quantifying physiological influences on otolith microchemistry. Methods Ecol. Evol. 2016;6:806-816.

9. Guerreiro PM, Costa R, Power DM. Dynamics of scale regeneration in seawater-and brackish water-acclimated sea bass, Dicentrarchus labrax. Fish Physiology and Biochemistry. 2013;39(4):917-930.

10. Gadomski DM, Mesa MG, Olson TM. Vulnerability to predation and physiological stress responses of experimentally descaled juvenile chinook salmon, Oncorhynchus tshawytscha. Environmental Biology of Fishes. 1994;39(2):191-199.

11. Zydlewski J, Zydlewski G, Danner GR. Descaling Injury Impairs the Osmoregulatory Ability of Atlantic Salmon Smolts Entering Seawater. Transactions of the American Fisheries Society. 2010;139(1):129-136.

12. Metz JR, Vrieze ED, Lock EJ, et al. Elasmoid scales of fishes as model in biomedical bone research. Journal of Applied Ichthyology. 2012;28(3):382-387.

13. Reist DJ, Halden MN, Loewen NT, et al. Linking physiology and biomineralization processes to ecological inferences on the life history of fishes. Comparative biochemistry and physiology, Part A. Molecular and integrative physiology. 2016;202:123-140.

14. Persson P, Johannsson SH, Takagi Y, et al. Estradiol-17 $\beta$ and nutritional status affect calcium balance, scale and bone resorption, and bone formation in rainbow trout, Oncorhynchus mykiss. Journal of Comparative Physiology B. 1997;167(7):468-473.

15. Bigler B. Mechanism and Occurrence of Focal Scale Resorption among Chum Salmon (Oncorhynchus keta) of the North Pacific Ocean. Canadian Journal of Fisheries and Aquatic Sciences. 2011;46(7):1147-1153.

16. Doherty AH, Ghalambor CK, Donahue SW. Evolutionary Physiology of Bone: Bone Metabolism in Changing Environments. Physiology. 2015;30(1):17-29.

17. Vrieze ED, Metz JR, Hoff JWVD, et al. ALP, TRAcP and cathepsin K in elasmoid scales: a role in mineral metabolism? Journal of Applied Ichthyology. 2010;26(2):210-213.

18. Vrieze ED, Sharif F, Metz JR, et al. Matrix metalloproteinases in osteoclasts of ontogenetic and regenerating zebrafish scales. Bone. 2011;48(4):704-712.

19. Vrieze ED, Heijnen L, Metz JR, et al. Evidence for a hydroxyapatite precursor in regenerating cyprinid scales. Journal of Applied Ichthyology. 2012;28(3):388-392.

20. Metz JR, Leeuwis RHJ, Zethof J, et al. Zebrafish (Danio rerio) in calcium-poor water mobilise calcium and phosphorus from scales. Journal of Applied Ichthyology. 2014;30(4):671-677.

21. Vrieze ED, Kessel M, Peters HM, et al. Prednisolone induces osteoporosis-like phenotype in regenerating zebrafish scales. Osteoporosis international: a journal established as result of cooperation between the European Foundation for Osteoporosis and the National Osteoporosis Foundation of the USA. 2014;25(2):567-578.

22. Bartel D P. MicroRNAs: Genomics, Biogenesis, Mechanism, and Function. Cell. 2004;116: 281-297.

23. Filipowicz W, Bhattacharyya SN, Sonenberg N. Mechanisms of post-transcriptional regulation by microRNAs: are the answers in sight? Nature Reviews Genetics. 2008;9(2):102-114.

24. Tanase CP. Molecular Pathology of Pituitary Adenomas || MicroRNAs. London, Elsevier. 2012:91-96.

25. Baumgart M, Barth E, Savino A, et al. A miRNA catalogue and ncRNA annotation of the short-living fish Nothobranchius furzeri. BMC Genomics. 2017;18(1):693.

26. Presslauer C, Bizuayehu TT, Kopp M, et al. Dynamics of miRNA transcriptome during gonadal development of zebrafish. Scientific Reports. 2017;7:43850.

27. Fantinatti BEA, Perez ES, Zanella BTT, et al. Integrative microRNAome analysis of skeletal muscle of Colossoma macropomum (tambaqui), Piaractus mesopotamicus (pacu), and the hybrid tambacu, based on next-generation sequencing data. BMC Genomics. 2021;22(1):237.

28. Desvignes T, Batzel P, Sydes J, et al. miRNA analysis with Prost! reveals evolutionary conservation of organ-enriched expression and post-transcriptional modifications in three-spined stickleback and zebrafish. Scientific Reports. 2019;9(1):3913.

29. Persson P, Takagi Y, Björnsson BT. Tartrate resistant acid phosphatase as a marker for scale resorption in rainbow trout, Oncorhynchus mykiss: effects of estradiol-17 $\beta$ treatment and refeeding. 1995;14(4):329-339.

30. Desjardin C, Vaiman A, Mata X, et al. Next-generation sequencing identifies equine cartilage and subchondral bone miRNAs and suggests their involvement in osteochondrosis physiopathology. BMC Genomics. 2014;15(1):798-811.

31. Sun J, Zhong N, Li Q, et al. MicroRNAs of rat articular cartilage at different developmental stages identified by Solexa sequencing. Osteoarthritis and Cartilage. 2011;19(10):1237-1245.

32. Shen Y, He DY. Research progress on signal transduction pathways regulating the differentiation and development of osteoclasts. Current Immunology. 2013;33(4):341-345+349 (in Chinese).

33. Lepetsos P, Papavassiliou KA, Papavassiliou AG. Redox and NF-KB signaling in osteoarthritis. Free Radical Biology and Medicine. 2018;132:90-100.

34. Zhang P, Liu J, Xu Q, et al. TLR2-dependent modulation of osteoclastogenesis by porphyromonas gingivalis through differential induction of NFATc1 and NF-KB. Journal of Biological Chemistry. 2011;286(27):24159-24169.

35. LIU Z, CHEN J, MIRANDO AJ, et al. A dual role for NOTCH signaling in joint cartilage maintenance and osteoarthritis. Science Signaling. 2015;8(386):ra71ra71.

36. Lin NY, Distler A, Beyer C, et al. Inhibition of Notch1 promotes hedgehog signalling in a HES1-dependent manner in chondrocytes and exacerbates experimental osteoarthritis. Annals of the Rheumatic Diseases, 2016;75(11):2037-2044.

37. Friedlnder MR, Mackowiak SD, Li N, et al. miRDeep2 accurately identifies known and hundreds of novel microRNA genes in seven animal clades. Nucleic Acids Research. 2012;40(1):37-52.

38. Westendorf JJ, Kahler RA, Schroeder TM. Wnt signaling in osteoblasts and bone diseases. Gene, 2004;341(1-2):19-39. 
39. Huang K, Fu J, Zhou W, et al. MicroRNA-125b regulates osteogenic differentiation of mesenchymal stem cells by targeting Cbf $\beta$ in vitro. Biochimie. 2014;102:47-55.

\section{Figures}

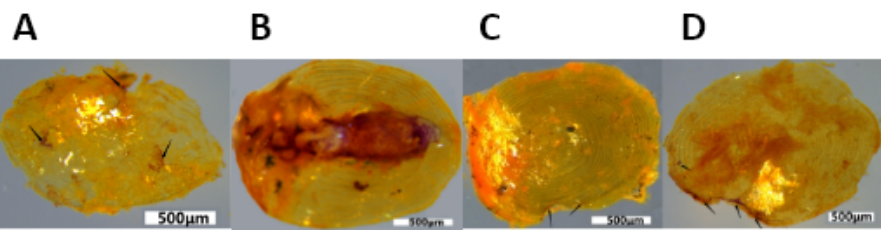

\section{Figure 1}

Representative examples of TRAcP staining on the scales of juvenile $O$. mykiss collected at different time points during salinity acclimation. Positive TRAcP staining sites were red (arrow heads), and most of the positive sites were located at the scale margins. (A): TRAcP dyeing results of scales from the fresh water group (CG); (B), (C) and (D): TRAcP staining for scales from seawater acclimation groups (7D, 14D and 21D, respectively); scale bar = $500 \mu \mathrm{m}$.

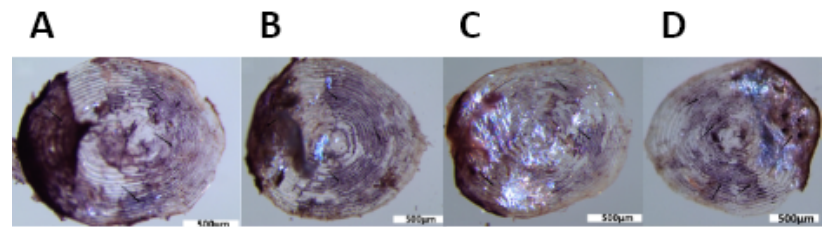

Figure 2

Representative examples of ALP staining on the scales of juvenile O. mykiss collected at different time points during salinity acclimation. Positive ALP staining sites were stained purple, and the positive sites were mainly located in the middle of scales. (A): ALP staining results of scales from the fresh water group (CG); (B), (C) and (D): ALP staining for scales from seawater acclimation groups (7D, 14D and 21D, respectively); scale bar = $500 \mu \mathrm{m}$.

\section{A \\ B C D}

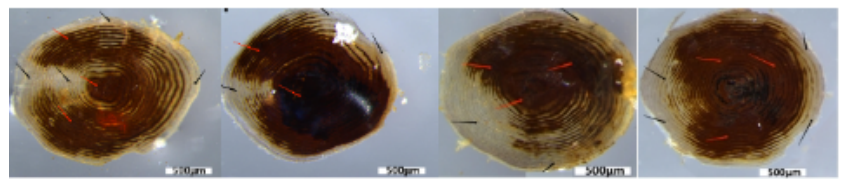

Figure 3

Representative examples of Von Kossa's staining on the scales of juvenile 0 . mykiss collected at different time points during salinity acclimation. Positive Von Kossa's staining sites (which stains the presence of calcium phosphates a brown colour) were mainly located in the middle of scales (red arrows); and unmineralized areas were mainly at the scale margins (black arrows). (A): Von Kossa's staining results of scales from the fresh water group (CG); (B), (C) and (D): Von Kossa's staining for scales from seawater acclimation groups (7D, 14D and 21D, respectively); scale bar $=500 \mu \mathrm{m}$.
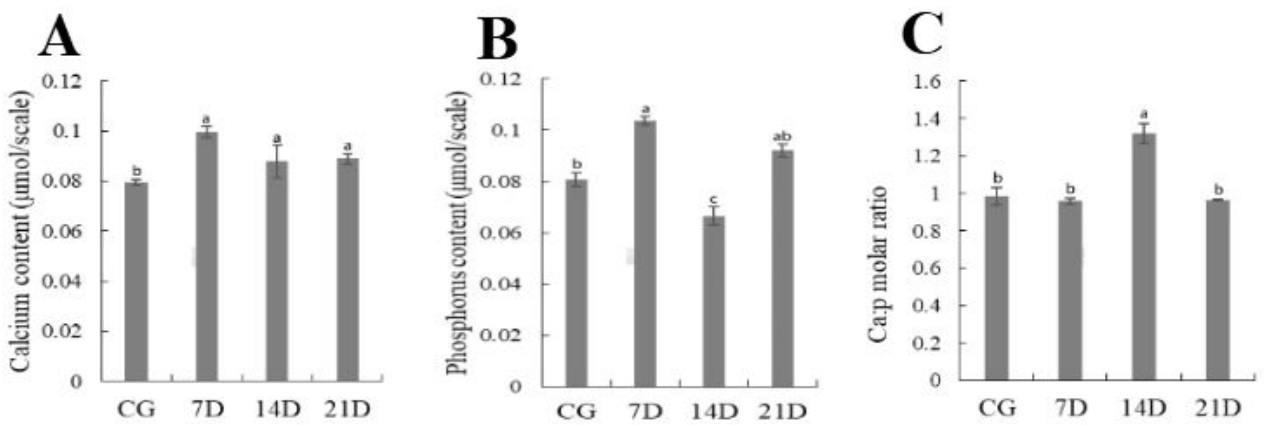

\section{Figure 4}

The calcium (Ca), phosphorus $(\mathrm{P})$ and calcium/phosphorus molar mass ratio $(\mathrm{Ca} / \mathrm{P})$ in scales of juvenile 0 . mykiss were respectively shown in (A), (B) and (C). CG represented freshwater control group; 7D, 14D and 21D respectively represented seawater acclimation of seven, 14 and 21 days. Data were expressed as means \pm standard deviations $(\nabla x \pm \mathrm{SD}) ; \mathrm{N}=24$ scales. Different letters represented significant difference $(P$-value $<0.05)$. 


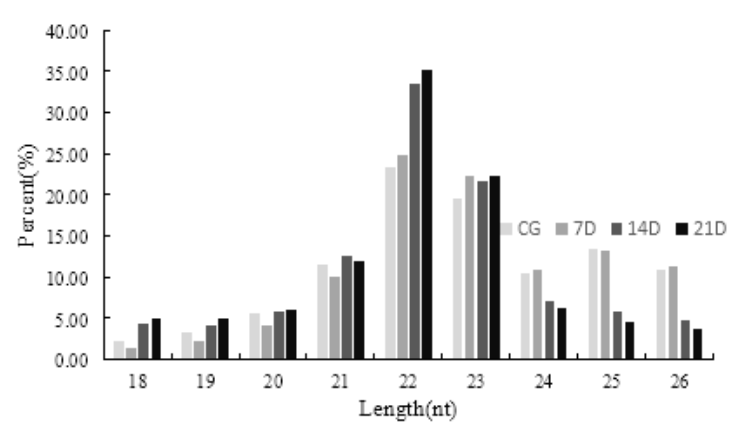

\section{Figure 5}

Length distribution of miRNAs found in juvenile O. mykiss scales collected at different time points during salinity acclimation.

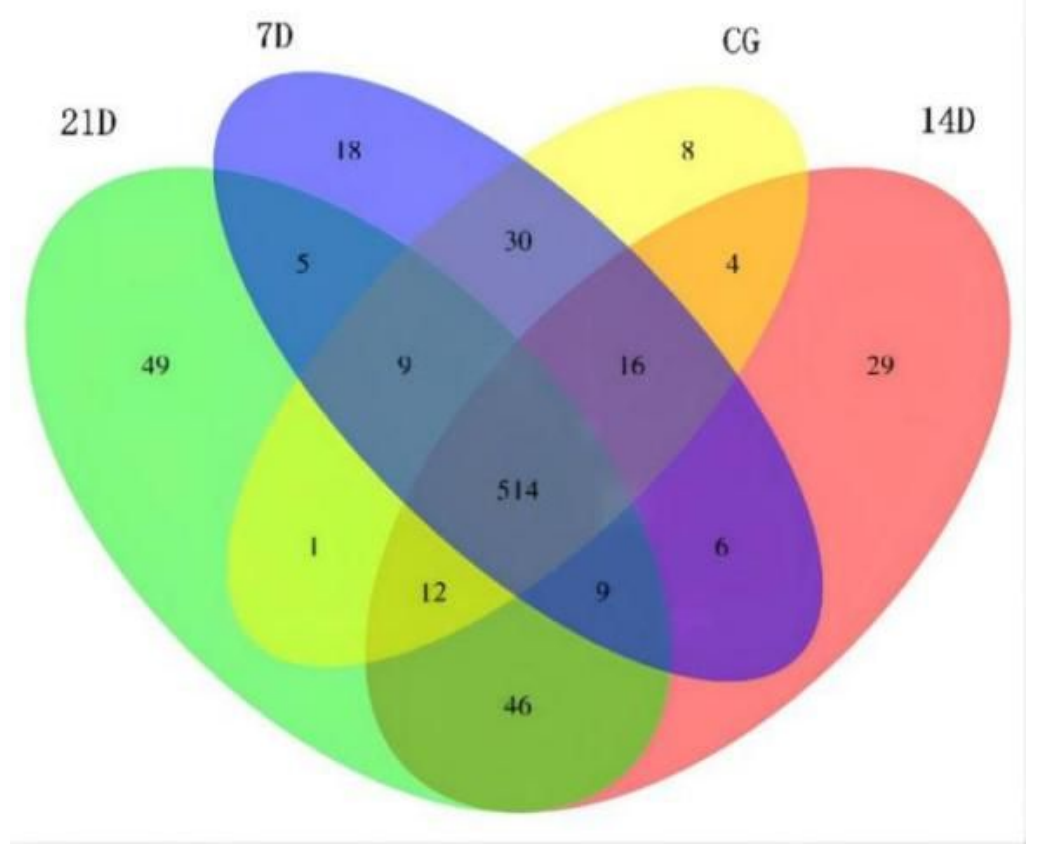

\section{Figure 6}

Venn diagram showing the number distribution of miRNAs expressed in 0 . mykiss scales collected at different time points during salinity acclimation. 

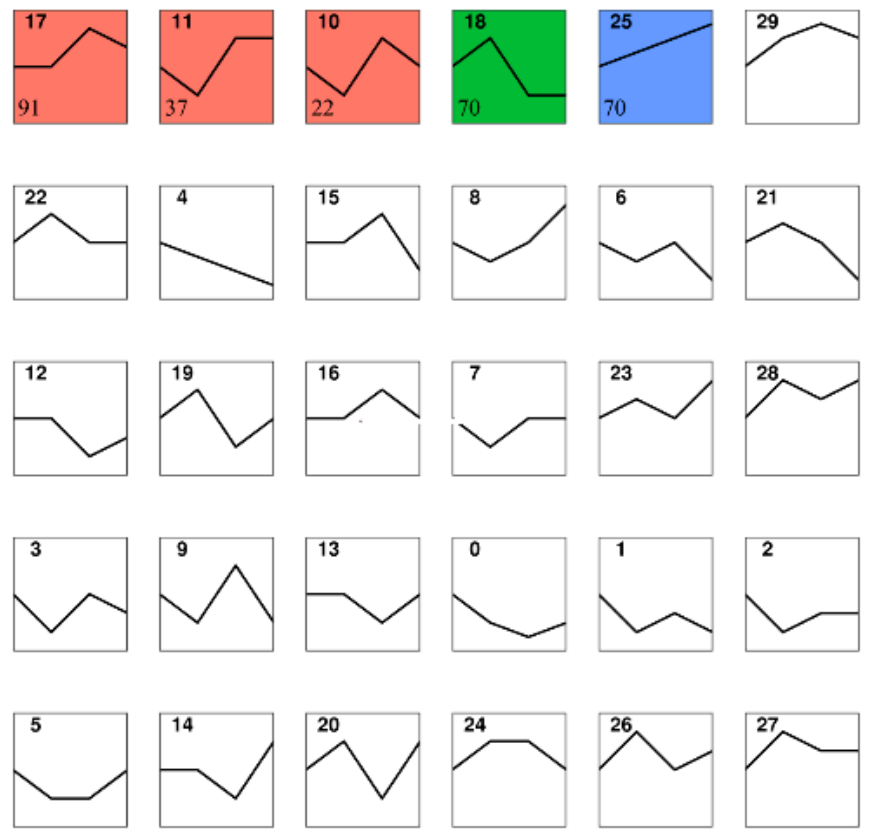

\section{Figure 7}

Sketchmap of the cluster analysis of DE miRNAs in scales of juvenile O. mykiss collected at different time points during salinity acclimation. Filled color in the clusters indicated significant trends $(P$-value $<0.05)$. The number at the lower left corner of the cluster represents the number of miRNAs in the cluster.
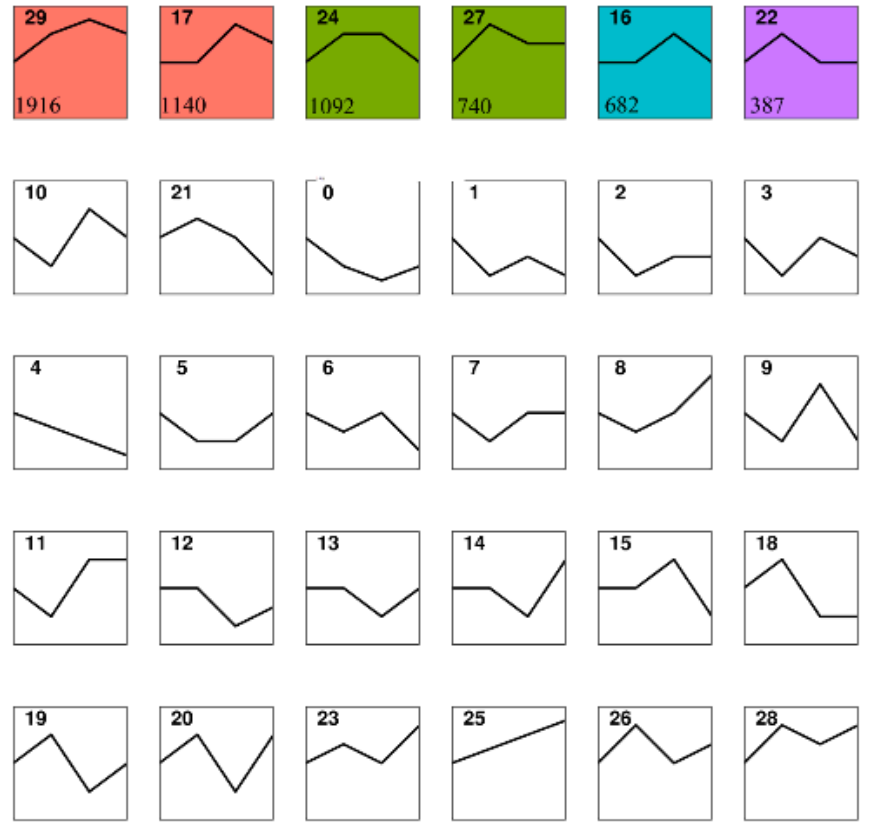

\section{Figure 8}

Sketchmap of the cluster analysis of DE genes in scales of juvenile $O$. mykiss collected at different time points during salinity acclimation. Filled color in the clusters indicated significant trends $(P$-value $<0.05)$. The number at the lower left corner of the cluster represents the number of genes in the cluster. 
A

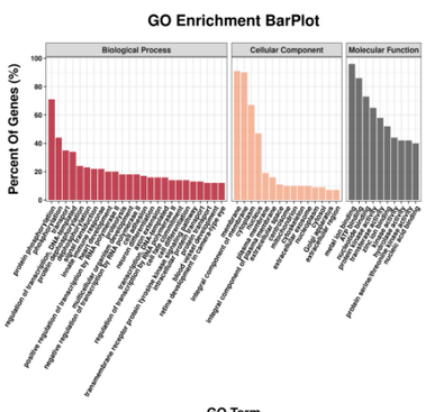

C

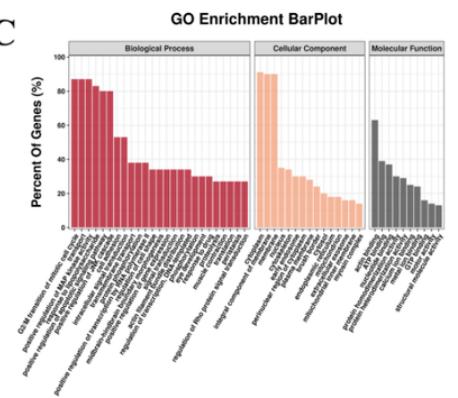

Go Term

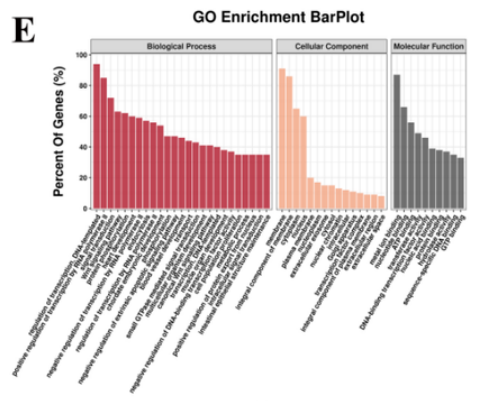

Go Term

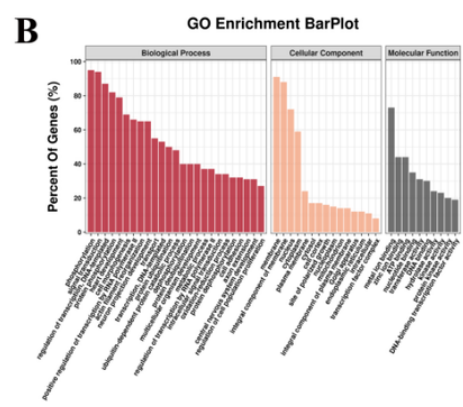

Go Term

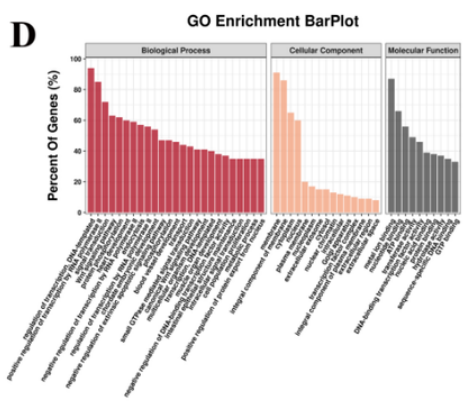

GO Term

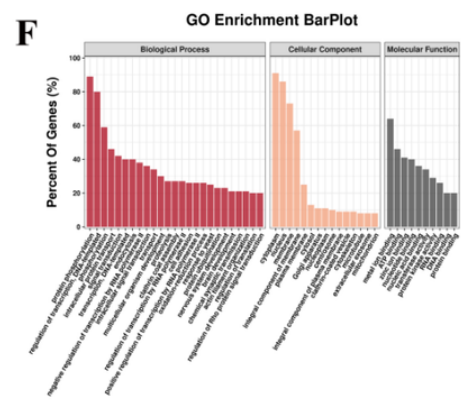

GO Term

\section{Figure 9}

DEC miRNA-targeted genes from clusters with significant trends in the cluster analysis based on gene ontology in scales of juvenile 0 . mykiss collected at different time points during salinity acclimation. Each color represents a categorie (A): Cluster 16; (B): Cluster 17; (C): Cluster 22; (D): Cluster 24; (E): Cluster 27; (F): Cluster 29. (According to the Fig. 8) 


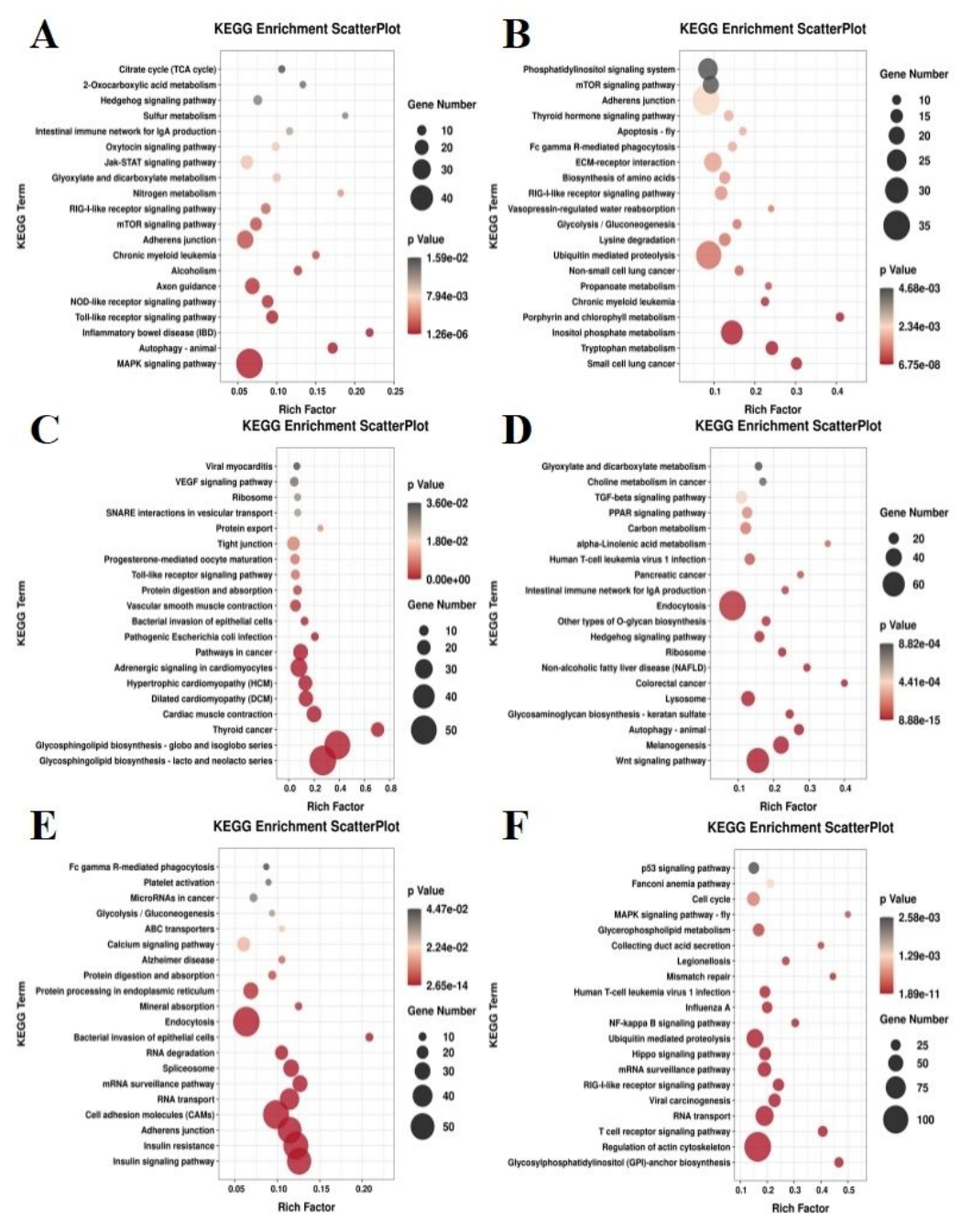

\section{Figure 10}

Pathway classifications of DEC miRNA-targeted genes from clusters with significant trends in the cluster analysis according to KEGG results of juvenile $O$. mykiss scales collected at different time points during salinity acclimation. Size of circle represented size of enriched DEC miRNA-targeted genes number. Shade of color represented size of P-value. (A): Cluster 16; (B): Cluster 17; (C): Cluster 22; (D): Cluster 24; (E): Cluster 27; (F): Cluster 29. (According to the Fig. 8)

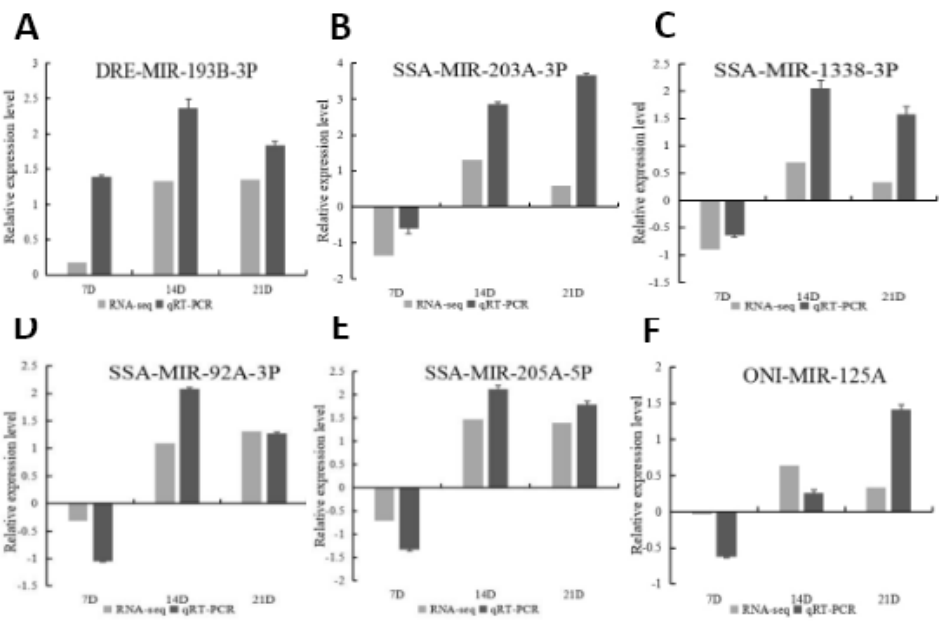

Figure 11

qRT-PCR verification of DE miRNAs. The relative expression levels of six miRNAs at 7D, 14D and 21D were calculated according to log ${ }_{2}$ (fold change) and

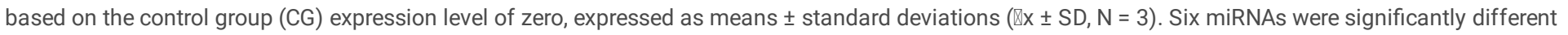


from CG at 7D, 14D and 21D ( $P$-value $<0.05)$.

\section{Supplementary Files}

This is a list of supplementary files associated with this preprint. Click to download.

- TableS1.DetailedinformationformiRNAidentificationandprediction.xIsx

- TableS2.DetailedinformationformiRNAfamilyidentification.xIsx

- TableS3.DetailedinformationfordifferentiallyexpressedDEmiRNAslog2FC1andp0.05.xlsx

- TableS4.DetailedinformationfordifferentiallyexpressedDEmiRNAtargetedgenesmRNAslog2FC1andp0.05.xlsx

- TableS5.DetailedinformationforthedifferentiallyenrichedGOtermanalysis.xIsx

- TableS6.DetailedinformationforthedifferentiallyenrichedKEGGpathways.xIsx 